Methods A retrospective chart review of all patients seen at the Anal Dysplasia Clinic at the Pittsburgh AIDS Center for Treatment was performed between 1 January 2008 and 30 June 2010. 41 HIV positive MSM had biopsy proven HGAIN (AIN1/2, AIN2, AIN 2/3, and AIN3) at high-resolution anoscopy. Treatment involved applying three sequential cotton swabs soaked in $80 \%$ TCA to the lesion. Patients were seen at an average of 6 months following treatment for follow-up. Clearance was defined as the absence of HGAIN epithelial markers at HRA (punctuation/mosaicism) or AIN $1 /$ normal epithelium on biopsy. The impact of the following variables on lesion clearance were assessed using univariate logistic regression analysis: age, CD4 count, initial grade of dysplasia on biopsy, concomitant therapy with imiquimod, previous history of HGAIN treatment.

Results The mean age of the study population was 52 years and the mean CD4 count was 565 cells/ml. Of the 43 HGAIN lesions treated in 41 patients, 22 (51\%) demonstrated clearance of HGAIN epithelial markers. Repeat biopsy was performed on eight lesions and 4 (9\%) lesions were AIN1, 3 (7\%) lesions were AIN2, and one lesion was ungradable. By univariate logistic regression analysis, patients with a diagnosis of AIN1/2 (compared with those who had AIN2 or greater) demonstrated a non significant trend towards clearance of abnormal visual markers (OR $3.8 \mathrm{p}=0.112$ ). All other predictive variables did not achieve statistical significance.

Conclusions On a per lesion basis, the success of a single visit triple topical application of TCA was $58 \%$ that is comparable with previously published treatment studies using techniques such as infrared coagulation. While larger prospective studies are called for, following exclusion of cancer by biopsy, HRA combined with TCA treatment may represent a low-cost, minimally invasive management strategy for this population with a high-incidence of HGAIN.

\section{P3-S4.05 MISSED OPPORTUNITIES FOR EARLY DETECTION OF CERVICAL CANCER IN AN STD CLINIC}

doi:10.1136/sextrans-2011-050108.466

J Arno, R Graffis, E Ferries-Rowe, L Hess. Indiana University School of Medicine, Indianapolis, USA

Background In 2009, more than 11000 women were diagnosed with invasive cervical cancer and approximately 4000 died. Many of these women were never screened or not screened in the 5 years before their diagnosis. Because vaccine uptake has been low, it is critical to continue efforts at early detection. We examined how many women diagnosed with cervical cancer at a public hospital were seen at the public STD clinic up to 5 years before diagnosis to detect missed opportunities for early detection.

Methods All cases with a diagnosis of cervical cancer from 1 January 1999 to 18 October 2010 were extracted from the electronic database of Wishard Hospital (Marion County Indiana's public hospital) using ICD-9 codes for severe squamous dysplasia (CIN3), adenocarcinoma in situ of the cervix and malignant neoplasm of cervix uteri. Results were validated using cytology and pathology records and women verified to have CIN3 or higher grade abnormalities were included. These were matched to patients in the electronic medical database of the Bell Flower Clinic (Marion County's public STD clinic) on the basis of first name, last name, date of birth, race and social security number when present. Missed opportunities were defined as those visits to Bell Flower within 5 years of a diagnosis of cervical cancer and no history of a PAP smear or HPV screening within 2 years at Wishard Hospital.

Results We identified 1309 women with cervical cancer diagnosed or treated at Wishard Hospital during the study period. Of these, 202 women were found to have visited Bell Flower at some time during the study period whereas 73 were found to have made 147 visits to Bell Flower Clinic $<5$ years prior to their diagnosis of CIN3 or higher. Because a substantial number of these women had normal or low grade abnormal PAPs at Wishard $<2$ years prior to their diagnosis, only 48 patients ( 97 visits) had visits that qualified as missed opportunities.

Conclusions Most women who developed cervical cancer diagnosed or treated at the public hospital had no record of a visit to the STD clinic. Of the 38813 visits by women to Bell Flower during the study period, only 147 visits qualified as missed opportunities for detecting cervical cancer in 48 individuals. The number of women affected by missed opportunities was low, in part, because of accessible strong PAP smears at the public hospital.

\section{P3-S4.06 MEN WITH GENITAL WARTS DO CONSULT LATER THAN WOMEN}

doi:10.1136/sextrans-2011-050108.467

P Dolcé, V Séguin, H Bernatchez. CSSS Rimouski-Neigette, Rimouski, Canada

Background CSSSRN is a regional hospital of 240 acute-care beds with a STD clinic which works mainly with clinical nurses, under the supervision of Infectious Diseases physicians (ID). This study aimed to review the clinical data of patients with genital warts.

Methods All files from patients with genital warts evaluated in our STD clinic between 2005 and 2010 were reviewed for clinical data including the delays before consultation. All data were analysed with Epi Info 3.5.2.

Results From 2005 to 2010, an average of 5950 patients per year attended our STD clinic, and 70\% were women. A total of 656 cases of genital warts were diagnosed by visual inspection from the attending ID physician. The specific types of lesions were warts associated with Human Papilloma Virus in $76 \%$, molluscum contagiosum $20 \%$, both $4 \%$. The median age was 24 years (range 16-76) and $57 \%$ of patients were men. The patients did not notice any lesion prior to consultation in 61 cases $(9.3 \%)$. For the others, the delays before consultation were: $0-7$ days $(16.2 \%) ; 8-14$ days (8.9\%); 15-27 days (12\%); 1-2 months (30\%); 3-5 months $(11.5 \%) ; 6-12$ months (11.9\%); >1 year (8.7\%). Significant longer delays before consultation were observed for men in comparison to women. The delays were: $\leq 7$ days (men 42/349 [12\%], women 54/ 244 [22.1\%], $\mathrm{p}<.001$ ); =14 days (men 70/349 [20.1\%], women 79/ 244 [32.4\%], $\mathrm{p}<.001$ ); $=27$ days (men 113/349 [32.3\%], women 107/ 244 [43.8\%], $\mathrm{p}=0.006$ ); $\geq 6$ months (men 90/349 [25.7\%], women $33 / 244$ [13.5\%], $\mathrm{p}<0.001$ ); more than 1 year (men $41 / 349$ [11.7\%], women $11 / 244$ [4.1\%], $\mathrm{p}=0.002$ ).

Conclusions In our population, the median delay before consultation for genital warts was 1-2 months. However, men with genital warts do consult significantly later than women. Specific public health approach should be considered for men with visible genital lesions, in order to promote earlier consultation and limit transmission. More behavioural studies are needed to further investigate this observation

\section{Clinical sciences poster session 5: other P3-S5.01 MICROBIAL DIVERSITY OF GENITAL ULCER DISEASE IN MEN ENROLLED IN A RANDOMISED TRIAL OF MALE CIRCUMCISION IN KISUMU, KENYA}

doi:10.1136/sextrans-2011-050108.468

${ }^{1} \mathrm{~S}$ Mehta, ${ }^{2}$ Maclain, ${ }^{1} \mathrm{R}$ Baiely, ${ }^{2} \mathrm{~S}$ Moses, ${ }^{3} \mathrm{P}$ Gillevet, ${ }^{4} \mathrm{G}$ Spear. ${ }^{1}$ University of Illinois, Chicago, USA; ${ }^{2}$ University of Manitoba, Winnipeg, Canada; ${ }^{3}$ George Mason University, USA; ${ }^{4}$ Rush University, USA

Background Medical male circumcision (MMC) reduces the risk of HIV acquisition by $\sim 60 \%$, in part by reducing genital ulcer disease 
(GUD) and HSV-2. In the Ugandan and Kenyan trials, PCR did not find a sexually transmissible agent in $50-60 \%$ of GUD specimens, and MMC did not protect against HSV-2 in the Kenyan trial. We sought to better define the aetiology of GUD in the Kenyan trial and examine how MMC affects GUD aetiology.

Methods We defined GUD of unknown aetiology as negative for HSV type 1 and type 2, T pallidum, and $H$ ducreyi by PCR, and negative for HSV-2 and T pallidum by serology. In 59 GUD specimens from 59 men, 23 (39\%) had unknown aetiology. We identified bacterial microbiota in all 59 specimens using multitag pyrosequencing of the $16 \mathrm{~S}$ rRNA gene, and compared results by unknown vs STI aetiology. Statistical analysis employed exact methods.

Results Overall, 83 distinct genera were detected. Prevotella spp. was most abundant, accounting for $18 \%$ of microbiota on average, and present in $75 \%$ of specimens. Bacterial diversity was greater in GUD of unknown aetiology than STI associated GUD (median number of genera 13 [range 7-20] vs 11 [range 3-20], $\mathrm{p}=0.06$ ). Fusobacterium spp., Sneathia spp. and Anaerococcus spp. were more abundant in GUD of unknown aetiology $(7.7 \%, 7.6 \%$, and $7.3 \%$, respectively) than in GUD of STI aetiology (4.6\%, 5.0\%, and $5.4 \%$ ). Fusobacteriales (Fusobacterium spp. or Sneathia spp.) $[\mathrm{OR}=4.7 ; 95 \%$ CI: 1.3 to 19.9] and Anaerococcus spp. [OR=4.6; 95\% CI: 1.2 to 22.5] were more likely to be recovered in GUD of unknown aetiology than STI associated GUD. Fusobacteriales were more often recovered from uncircumcised men than circumcised men (62\% vs $22 \%$, $\mathrm{p}=0.04$ ), and Anaerococcus spp. was present in $22 \%$ of circumcised vs $70 \%$ of uncircumcised men $(p=0.010)$. Reported penile coital injuries were more common among men with Anaerococcus spp. $(85 \%$ vs $57 \%, p=0.01$ ), and condom use was less common ( $50 \%$ vs $71 \%$, $\mathrm{p}=0.11$ ). There was no difference in these bacteria by ulcer location. Conclusions Fusobacteriales and Anaerococcus spp. may colonise genital ulcers that develop from a mechanism related to circumcision status. Many such "ulcers" may be epithelial disruptions that are traumatic in origin. These bacteria have cytotoxic properties that may ulcerate or exacerbate pre-existing minor epithelial disruptions. MMC may reduce GUD through a reduction in these anaerobic bacteria.

\section{P3-S5.02 IS URETHRAL SMEAR MICROSCOPY IN ASYMPTOMATIC MEN EFFECTIVE IN REDUCING MAJOR M GENITALIUM INFECTION SEOUELAE IN WOMEN?}

doi:10.1136/sextrans-2011-050108.469

${ }^{5} \mathrm{P}$ White, ${ }^{1} \mathrm{R}$ Birger, ${ }^{2} \mathrm{~J}$ Saunders, ${ }^{2} \mathrm{C}$ Estcourt, ${ }^{1} \mathrm{~T}$ Hallett, ${ }^{3} \mathrm{O}$ Caffrey, ${ }^{4} \mathrm{C}$ Mercer, ${ }^{3,6} \mathrm{~T}$ Roberts. ${ }^{1}$ Imperial College, School of Public Health, London, UK; ${ }^{2}$ St Bartholomew's Hospital, London, UK; ${ }^{3}$ University of Birmingham, Birmingham, UK; ${ }^{4}$ University College London, London, UK; ${ }^{5}$ Health Protection Agency, London, UK; ${ }^{6}$ UK

Background Non-Chlamydial Non-Gonococcal Urethritis (NCNGU) is as prevalent as Chlamydial urethritis in UK men. Mycoplasma genitalium likely explains $10-46 \%$ of cases but there is no UKlicensed molecular test. In women, $M$ genitalium is associated with pelvic inflammatory disease (PID), infertility and ectopic pregnancy. New UK guidelines do not recommend urethral smear microscopy in asymptomatic men, so asymptomatic NCNGU cannot be diagnosed, whereas previously affected men and their sexual partners were treated with antibiotics. The impact of this policy on reproductive health sequelae in women is unknown.

Methods We used a compartmental mathematical model to synthesise evidence on the natural history of $M$ genitalium, diagnosis and treatment levels. Uncertainty was accounted-for using Latin hypercube sampling, with rejection sampling to fit to data. The model was stratified by sex, incorporates heterogeneous sexual behaviour, and was parameterised by behaviour data from key UK surveys (NATSAL2, PATSI, MSTIC); STI surveillance data; and natural history data from the literature. It incorporates symptomatic and asymptomatic infection; PID; care-seeking due to symptoms, partner notification, and routine screening; and treatment failure. We fitted to national STI surveillance (KC60) data, (allowing for uncertainty in the amount of NCNGU caused by $M$ genitalium), representing current practice, and examined (i) increasing microscopy in asymptomatic men by making it routine in all genito-urinary medicine clinics and (ii) decreasing rates by discontinuing it in the $20 \%$ of clinics currently still using it.

Results Microscopy in asymptomatic men reduces the burden of infection in women by reducing prevalence in men thus reducing transmission to women; and treating women via partner notification from diagnosed men. The magnitude of these effects depends upon natural history in men and women, about which there is uncertainty. We determine ranges of parameter values in which routine microscopy would be effective in reducing $M$ genitaliumrelated sequelae in women.

Conclusions Further empirical work is required to determine if natural history parameters fall in the ranges that would make routine microscopy in asymptomatic men effective. Particularly important are the prevalence of $M$ genitalium; proportion of infections that are symptomatic in men and women; duration of untreated infection; and incidence of PID, infertility and ectopic pregnancy attributable to $M$ genitalium.

\section{P3-S5.03 CONFLICT OF INTEREST AND POINT OF CARE TESTS: AN EXPLORATION OF ACCURACY IN HEPATITIS C INFECTION}

doi:10.1136/sextrans-2011-050108.470

${ }^{1} \mathrm{~S}$ Shivkumar, ${ }^{1} \mathrm{Y}$ Jafari, ${ }^{2} \mathrm{G}$ Lambert, ${ }^{2} \mathrm{C}$ Claessens, ${ }^{1} \mathrm{M}$ Klein, ${ }^{3} \mathrm{~J}$ Martinez-Cajas ${ }^{4} \mathrm{R}$ Peeling, ${ }^{1} \mathrm{~L}$ Joseph, ${ }^{1} \mathrm{~N}$ P Pai. ${ }^{1}$ McGill University, Montreal, Canada; ${ }^{2}$ INSPQ, Canada; ${ }^{3}$ Queen's University, Canada; ${ }^{4}$ LSHTM, UK

Background The WHO estimates that 170 million people worldwide are infected with Hepatitis C. In the context of HIV co-infection, rapid point-of-care tests gain importance in both the developing and developed countries. Moreover, in the light of the Food and Drug Administration's approval of the Oraquick point-of-care test for Hepatitis C for use in the USA, the accuracy of these tests is relevant.

Objective We conducted a systematic review and meta-analysis of the literature examining the sensitivity and specificity of all rapid point-of-care tests used to diagnose incident or prevalent Hepatitis $\mathrm{C}$, with an attention to involvement of industry in reporting of results.

Methods Two reviewers conducted independent searches of five databases between the years of 1995 and 2010. Bayesian metaanalysis was conducted accounting for the use of imperfect reference standards (sensitivity and specificity ranges of $90 \%-100 \%$ were assumed) in the assessment of index tests. The quality of all included full-text studies was assessed using the QUADAS and STARD checklists, with a focus on reporting of conflict of interest with industry.

Results A total of seven studies were identified from the database searches, of which five were conducted in developing settings. Eight index tests were examined including Oraquick, HCV Tri-Dot, HCV Bidot, Therma Ricerca, SM-HCV, Onecheck, Goldspot and Accurate. Sensitivity of all index tests ranged from $45 \%$ to $100 \%$, while specificity ranged from $93 \%$ to $100 \%$. Oraquick reportedly had the highest accuracy, with sensitivity ranging from $99 \%$ to $100 \%$ and a specificity of $100 \%$. However, the authors of the study reported a financial relationship with Orasure Technologies Inc., the makers of Oraquick. Although pooled sensitivity of all tests was high at 